\title{
Los DeRECHOS Humanos EN El CAMPo MÉdico: UNA POSTURA JURÍDICA
}

human Rights in The Medical field: A Legal Position'

Carolina Campos Serrano²; Laura PÁez díaz de León ${ }^{3}$

Resumen: La complejidad de la práctica médica en hospitales ocasiona se vulnere el derecho humano a la salud. A partir de la observación del comportamiento de los médicos más jóvenes quienes se encuentran en proceso de formación profesional y de aquéllos que cursan la residencia médica especialización - en estudios de posgrado, es que en este artículo se muestra como las nuevas generaciones de médicos son propensos a transgredir derechos humanos y cometer delitos por el desconocimiento de las consecuencias de sus actos. Se plantea la necesidad de que los derechos humanos sean parte de la educación en el campo médico y la urgencia de crear un modelo jurídico basado en derechos humanos y legalidad que permita armonizar la educación del área médica con el derecho.

Palabras Glaves: Derechos humanos, derecho a la salud, campo médico, educación en derechos humanos.

Abstract: The complexity of medical practice in hospitals

${ }^{1}$ El presente trabajo se plantea en el marco de la estancia de investigación posdoctoral. Se agradece al Programa de Becas Posdoctorales de la Dirección General de Asuntos del Personal Académico UNAM el apoyo brindado para efectuar la presente investigación.

${ }^{2}$ Doctora en Derecho, profesora investigadora adscrita a la Facultad de Estudios Superiores Acatlán, UNAM. México. Contacto: <caritocamposs@gmail.com>. ORCID:

${ }^{3}$ Doctora en Letras Modernas, Profesora de Tiempo Completo Definitiva y secretaria de Investigación y Posgrado de la Facultad de Estudios Superiores Acatlán, UNAM. México. Contacto: <laurapaez.unam@gmail.com>. ORCID:

Fecha de recepción: 20 de noviembre de 2020; fecha de aprobación: 14 de octubre de 2021 . 
causes the human right to health to be violated. Based on the observation of the behavior of the youngest doctors who are in the process of professional training and those who are studying medical residency - specialization - in postgraduate studies, this article shows how the new generations of doctors are prone to transgressing human rights and committing crimes due to ignorance of the consequences of their actions. There is a need for human rights to be part of education in the medical field and the urgency of creating a legal model based on human rights and legality that allows harmonizing education in the medical field with the law.

KeYwORDs: Human rights, right to health, medical field, human rights education.

Sumario: I. Preámbulo; II. Consideraciona es sobre los conceptos y su vinculación teórica; III. Metodología; IV. Evidencia empírica; V. Hallazgos; VI. Conclusiones.

\section{Preámbulo}

1. Planteamiento del problema

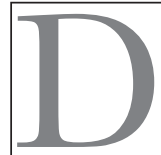

esde principios del siglo XXI el problema de la mala atención proporcionada por el personal de la salud ha llamado especialmente la atención. Sin embargo, el enfoque que las autoridades de salud observan es que aquél va encaminado a un problema en la calidad en los servicios. ${ }^{4}$ Por este problema muchos más se han evidenciado, entre ellos se destaca el de la violencia obstétrica que reveló por diversos medios de comunicación, como los derechos reproductivos de las mujeres son fuertemente transgredidos. ${ }^{5}$

${ }^{4}$ Hernández Torres, Francisco. La calidad de la atención a la salud en México a través de sus instituciones: 12 años de experiencia. Coordinado por Martha Alicia Alcántara Balderas. Secretaría de Salud. México, 2012. p.12

${ }^{5}$ El periodista Pedro Matías realizó un reportaje especial para la revista Proceso 
La presente investigación se plantea en el marco de la estancia posdoctoral realizada en la Universidad Nacional Autónoma de México con el proyecto el derecho humano a la salud y su estudio en las prácticas obstétricas. Así, iniciada la investigación se encontró que existen muchos estudios oficiales por autoridades ${ }^{6}$, academia ${ }^{7}$ y por asociaciones privadas ${ }^{8}$ que visibilizan la gran problemática que existe en el ámbito del derecho a la salud. La Comisión Nacional de los Derechos Humanos a solicitud de recomendaciones internacionales ${ }^{910}$ ha tomado como suya esta problemática para investigarla y contribuir para aminorarla, un ejemplo de ello es la "recomendación general 31/2017. Sobre la violencia obstétrica en el Sistema Nacional de Salud" que pronunció ese organismo autónomo. ${ }^{11}$

publicado el 4 de octubre de 2013, el cual en su título decía "Irma, la indígena mazateca que parió en el patio de un hospital", lo mostraba gráficamente lo cual indigno al país. Derivado de esta y otras publicaciones en los medios de comunicación es que la CNDH atrae el caso y emite la Recomendación No. 1/2014 sobre el caso de la inadecuada atención médica de $\mathrm{V} 1$ y su recién nacido V2, indígenas mazatecos, en el centro de salud rural del municipio San Felipe Jalapa de Díaz, Oaxaca, emitida el 29 de enero de 2014 por el presidente de la CNDH, Dr. Raúl Plascencia Villanueva.

6 "Diagnóstico sobre victimización a causa de violencia obstétrica en México", Comisión Ejecutiva de Atención a Víctimas, 2017. Disponible en: https://www.gob. mx/cms/uploads/attachment/file/194701/Diagno_stico_VO_port.pdf

${ }^{7}$ Castro Pérez, Roberto, "Génesis y práctica del habitus médico autoritario en México". Revista Mexicana de Sociología 76, núm. 2, abril-junio, 2014

${ }^{8}$ GIRE, Informe "Omisión e Indiferencia. Derechos Reproductivos en México", Capítulo 4. Violencia Obstétrica, Grupo de Información en Reproducción Elegida, AC, México, 2013.

${ }^{9}$ Como es el caso de México ante la Convención sobre la eliminación de todas las formas de discriminación contra la mujer, CEDAW por sus siglas en inglés, que nuestro país rinde informes sobre los avances para erradicar la violencia contra las mujeres.

${ }^{10}$ Recomendaciones Internacionales a México en materia de Derechos Humanos. Disponible en: http://recomendacionesdh.mx/

${ }^{11}$ La síntesis de dicha recomendación general fue publicada en el Diario Oficial de la Federación el miércoles 30 de agosto de 2017 en donde señala como objeto el contribuir a que las autoridades de salud identifiquen y combatan aquellas violaciones a derechos humanos de las mujeres en ocasión de la atención obstétrica. 
No obstante, en estos últimos años el sector salud ha estado en constante movimiento $^{12}$. Evidencia de ello fue que durante el mes de abril de 2019, el entonces director del IMSS, Germán Martínez Cázares — director a partir del 3 de diciembre 2018-, aceptó el grave problema que existe en ese instituto y aseguró que todos esos males son heredados por administraciones pasadas: La falta de presupuesto y la consecuente carencia de equipos, instalaciones adecuadas y personal calificado en varias áreas tienen al Instituto Mexicano del Seguro Social en los bajos niveles de cobertura y de calidad de su historia ${ }^{13}$. Ante esta problemática que vive el Seguro Social se exhibe la existencia de la responsabilidad y violencia institucional del Estado Mexicano hacia todo el personal médico que presta el servicio de salud. Aunado a lo anterior también señaló, el entonces director del IMSS que existen fraudes y robos tanto de recetas médicas como en medicamentos y de recursos materiales: La corrupción también está en el IMSS.

Se agrava la problemática cuando los medios de comunicación dan noticias donde señalan que la negligencia médica, por parte de algunos médicos, han ocasionado la muerte de algunas personas. ${ }^{14}$ Aun cuando por otro lado, también cabe apuntar que se han mostrado las capacidades médicas que tienen los médicos comprometidos con su profesión, quienes aun sin tener todo el equipo necesario

${ }^{12}$ Se refiere específicamente a los años comprendidos entre el 2018 al 2020. Años en que hubo recorte presupuestal para el sector salud, se reformó al artículo 4 constitucional en el contexto al derecho a la salud y a su ley reglamentaria, se creó el Instituto del Bienestar y el contagio por la pandemia derivada del coronavirus SARS Cov2 que ha puesto en jake a los sistemas de salud en el mundo.

${ }^{13}$ Gobierno de México. \#IMSS en ruinas: German Martínez, video, revista panorama, México, 8 de abril de 2019. Ver en Línea. URL: https://youtu.be/7788nj89Rc /

${ }^{14}$ Bañuelos, Claudio, Investigan muerte de madre y su bebe por negligencia en el ISSSTE. La Jornada. 15 noviembre de 2019. Disponible en: https://www.jornada. com.mx/ultimas/sociedad/2019/11/15/por-negligencia-muere-madre-y-su-bebeen-el-issste-de-aguascalientes-8551.html 
para trabajar, salvan las vidas de los pacientes. ${ }^{15}$

Los problemas que se presentan en el sector salud ${ }^{161718}$ son muy graves, los cuales han crecido desordenadamente y ahora se ha convertido en una problemática gigantesca que no se puede resolver en lo individual porque están interrelacionados con otros factores que a su vez dan vida a otros problemas.

Del mismo modo, existen investigaciones bajo una mirada sociológica en la que se ha argumentado que el problema de deshumanización en el sector salud se debe a la instrucción recibida en sus primeros años de estudiantes de pregrado en donde adquieren la formación de médico. A esta formación es lo que han denominado el Habitus Medico. ${ }^{19}$ Este habitus médico se conforma por tres características principales: 1. El entrenamiento de la incertidumbre, para reconocer el conocimiento y distinguirlo con las limitaciones propias. 2. El entrenamiento para la atención distante, en el que se enseña a no involucrarse moralmente con los pacientes, y 3. Entrenamiento para el "manejo de los errores y fracasos" en su práctica, donde se enseña al desapego total a los pacientes en el caso de error. ${ }^{20}$ Además de las anteriores existen otras prácticas más, que se

${ }^{15}$ Comunicado del IMSS. Médicos salvan a bebé tras realizar cesárea a su mamá en estado de coma. W Radio, ciudad de México, 17 de junio de 2019. Disponible en: https://wradio.com.mx/radio/2019/06/17/nacional/1560799796_173288.html

${ }^{16}$ Benassini, Oscar. "Atención en salud mental regionalización y reorientación en el marco de la descentralización de servicios de salud en México. "Salud Mental 20.4 (1997): 48-53.

${ }^{17}$ Dreser, Anahí, et al. "Uso de antibióticos en México: revisión de problemas y políticas." salud pública de México 50 (2008): S480-S487.

${ }^{18}$ Híjar-Medina, Martha, María Victoria López-López, and Julia Blanco-Muñoz. «La violencia y sus repercusiones en la salud: Reflexiones teóricas y magnitud del problema en México.» salud pública de México 39 (1997): 565-572.

${ }^{19}$ Castro Roberto y Erviti, Joaquina. (2015) Sociología de la práctica médica autoritaria. Violencia obstétrica, anticoncepción inducida y derechos reproductivos. UNAM, Cuernavaca, 2015, p. 51

${ }^{20}$ Ibidem, p. 55 
desarrollan durante su formación en los hospitales escuelas, llamado el currículo oculto. 1) El Disciplinamiento Sistemático, consistente en una forma de castigos que obligan a estudiar a los estudiantes en caso de errores. 2) Las jerarquías dentro de la profesión, donde se enseña a respetar aquéllos que tienen mayores conocimientos. Respeto a sus superiores. 3) La distinción de género, se alude a aquéllos que no son aptos para desarrollarse en determinadas áreas del campo de la salud. ${ }^{21}$

El habitus médico permea no solo entre los médicos, sino también lo hace hacia el personal de enfermería e incluso entre el personal administrativo. Todos ellos son influenciados por ese habitus generándose conductas de autoritarismo y arbitrariedades entre ellos mismos y dirigiéndose también hacia los pacientes y familiares de los pacientes ${ }^{22}$. Esto se ha traducido en una deshumanización para aquéllos que son ajenos a ese medio.

Además, se observa que la violencia que vive la sociedad mexicana $^{23}$ ha permeado en todo el entorno, por lo que es imposible pensar que un hospital pueda permanecer ajeno a estas dinámicas sociales. Los hospitales las manifiestan a través de la violencia insti-

${ }^{21}$ Ibidem, p. 59

${ }^{22}$ Ibidem, p. 62-64

${ }^{23}$ Nájar, Alberto, Violencia en México: cómo se explica el nuevo récord en el número de homicidios, BBC News Mundo, México, 22 julio 2019. Disponible en: https://www.bbc. com/mundo/noticias-america-latina-49079323 
tucional, ${ }^{24}$ la violencia obstétrica ${ }^{25}$, la violencia laboral ${ }^{26}$ e incluso la violencia por robo $^{27} \mathrm{u}$ homicidio a mano armada ${ }^{28}$.

La investigación documental ${ }^{29}$ no pudo resolver todas las interrogantes, por ello a través de la investigación de campo se buscó corroborar que a) el habitus médico sigue siendo el mismo o se ha modificado, b) que la violencia institucional conduce a provocar conductas deshumanizadas y c) que la generación demográfica Millennials es la constante en la que se señalan las principales negligencias médicas.

${ }^{24}$ De acuerdo con la Ley General de Acceso de las Mujeres a una Vida Libre de Violencia, son los actos u omisiones de las y los servidores públicos de cualquier orden de gobierno que discriminen o tengan como fin dilatar, obstaculizar o impedir el goce y ejercicio de los derechos humanos de las mujeres, así como su acceso al disfrute de políticas públicas destinadas a prevenir, atender, investigar, sancionar y erradicar los diferentes tipos de violencia.

${ }^{25}$ La Secretaría de Salud define la Violencia Obstétrica como una forma específica de violación a los derechos humanos que implica toda acción u omisión por parte del personal del sistema de salud, que dañe, lastime o denigre a las mujeres durante el proceso preconcepcional, el embarazo, parto/nacimiento y puerperio, en "Guía de Implantación. Modelo de atención a las mujeres durante el embarazo, parto y puerperio. Enfoque Humanizado, Intercultural y Seguro”, SSA: México, 2012, p. 95

${ }^{26}$ A quien obstaculice o condicione el acceso de una mujer a un empleo, por el establecimiento de requisitos referidos a su sexo, edad, apariencia física, estado civil, o condición de madre estará incurriendo en violencia laboral, tal y como se prevé en el artículo 279 del código penal del estado de México.

${ }^{27}$ IMSS denuncia robo de medicamentos controlados, en su comunicado oficial No. 106/2019 del mes de mayo de 2019 publicado en su página oficial donde señaló que el robo fue de 191 piezas de 14 medicamentos distintos de la farmacia del Hospital General de Zona (HGZ) No. 29, ubicado en la colonia San Juan de Aragón, de la Alcaldía de Gustavo A. Madero

${ }^{28} \mathrm{El}$ Universal. Con camioneta, derriban portón para rematar a hombre en hospital de Guanajuato. El Universal, redacción, sección Estados, ciudad de México, junio de 2018, Disponible en: https://www.eluniversal.com.mx/estados/con-camioneta-derriban-porton-para-rematar-hombre-en-hospital-de-guanajuato

${ }^{29}$ Muñoz Rocha, Carlos, "Metodología de la investigación”, Oxford, México, 2015, pp. 287 y 288 
Cabe aclarar que esta investigación se concluyó antes de que estuviera presente el aislamiento ${ }^{30}$ en torno al coronavirus SARSCoV-2 conocido como Covid- $19^{31}$ y se modificaran las condiciones en la atención médica en los hospitales.

La investigación teórica ${ }^{32}$ apoyada en la técnica documental arrojo los siguientes resultados:

i. Desconocimiento de la disciplina de Derechos Humanos.

ii. Desconocimiento de la Responsabilidad Jurídica en que se incurre por una conducta contraria a derecho.

iii. Necesidad urgente de impartir un Taller de Sensibilización de DDHH a estudiantes de Pregrado y de Posgrado en las instituciones públicas de salud.

iv. El Estado Mexicano incumple sus obligaciones en materia de derechos humanos en el sector salud.

${ }^{30}$ Aun cuando la Fase 2 de la contingencia sanitaria por el coronavirus SARSCoV2 - COVID-19, fue anunciada por el Gobierno de México el martes, 24 de marzo de 2020, múltiples instancias gubernamentales y privadas se anticiparon a la suspensión de actividades desde el 17 de marzo, de manera parcial o total, para evitar el contagio en el país.

${ }^{31}$ El coronavirus SARS-CoV-2 es un nuevo tipo de coronavirus que puede afectar a las personas y que se detectó por primera vez en diciembre de 2019 en la ciudad de Wuhan, provincia de Hubei, en China. Mayoritariamente, en un 80\% de los casos solo produce síntomas leves respiratorios. El virus se conoce como Coronavirus SARSCoV-2 y la enfermedad que causa se denomina COVID-19, en ¿Qué es el Coronavirus SARS-CoV-2? Hospital Clínic de Barcelona, Universidad de Barcelona. Disponible en: https://www.clinicbarcelona.org/asistencia/enfermedades/covid-19/definicion

${ }^{32}$ La investigación teórica identifica alguna hipótesis que descansa en una teoría. Ésta se confirma o se modifica de acuerdo con los resultados obtenidos al tratar de comprobar la hipótesis, en Schmelkes, Corina y Schmelkes, Nora, "Manual para la presentación de anteproyectos e informes de Investigación", Oxford, tercera edición, México, 2012, p.54 
II. Consideraciones Sobre los conceptos Y SU Vinculación TEÓRICA

Para la investigación empírica se plantearon los conceptos derechos a la salud, a la igualdad y discriminación y su vinculación con otros derechos humanos bajo la óptica de la teoría de los derechos humanos.

La teoría de los derechos humanos "se sostiene en una serie de instrumentos jurídicos diseñados por representantes de las naciones y se complementa con el trabajo de instancias investigadoras, conciliadoras y jurisdiccionales especializadas que evalúan su cumplimiento". Esta teoría sustenta que "Todos los seres humanos nacen libres e iguales en dignidad y derechos y, dotados como están de razón y conciencia, deben comportarse fraternalmente los unos con los otros." "34

Los derechos humanos se rigen por cuatro principios rectores: Principio de Universalidad, que implica el reconocimiento de todos los derechos en favor de todas las personas, independientemente de su posición jurídica ${ }^{35}$. El Principio de Interdependencia, el cual apela a la correlación que existe entre todos los derechos, de modo que el cumplimiento de uno promueve y facilita el cumplimiento de los demás y, por lo contrario, el obstáculo o negación de uno implica el de otro u otros ${ }^{36}$. El Principio de Indivisibilidad, radica en que

${ }^{33}$ Hernández Barrón, Alfonso. (2016) La teoría jurídica de los derechos humanos; el orden que surge del caos. Perspectiva desde el trabajo de la corte interamericana de derechos humanos. Tesis doctoral dirigida por José Luis Piñar Mañas. España: Universidad CEU San Pablo. Disponible en: https://dialnet.unirioja.es/servlet/tesis?codigo $=118416$

${ }^{34}$ Declaración Universal de los derechos humanos, Art. 1.

${ }^{35}$ Vázquez, Luis Daniel y Serrano, Sandra. (2011) Los principios de universalidad, interdependencia, indivisibilidad y progresividad. Apuntes para su aplicación práctica en "La reforma constitucional de derechos humanos: un nuevo paradigma", Coords. Pedro Salazar Ugarte y Miguel Carbonell Sánchez, México, Instituto de Investigaciones Jurídicas, UNAM, p. 140

${ }^{36}$ Vázquez, Luis Daniel y Serrano, Sandra. (2011) Los principios de universalidad, inter- 
los derechos forman en su conjunto un corpus indivisible, es decir, no se puede otorgar uno o unos y negar otro u otros. Asimismo, se refiere a que el ejercicio o goce de los derechos acarrea la obtención de todas las prestaciones que el mismo otorga y no es posible respetar o garantizar únicamente una o unas de esas prestaciones, y negar el resto. No existe jerarquía entre unos y otros ${ }^{37}$; y el Principio de Progresividad, que atiende a la idea de la expansión de la protección y del beneficio a todas las personas y, a su vez, a la ampliación de la obligación protectora, promotora, garante y respetuosa de la autoridad en la materia ${ }^{38}$.

Así, si se atiende a la correlación que existe entre todos los derechos, de modo que el cumplimiento de uno promueve y facilita el cumplimiento de los demás, entonces es posible entender que cuando se viola el derecho a la salud ${ }^{39}$, también a la par se están violando otros derechos.

El concepto de Salud se define como un estado de completo bienestar físico, mental y social, y no solamente la ausencia de

dependencia, indivisibilidad y progresividad. Apuntes para su aplicación práctica en "La reforma constitucional de derechos humanos: un nuevo paradigma", Coords. Pedro Salazar Ugarte y Miguel Carbonell Sánchez, México, Instituto de Investigaciones Jurídicas, UNAM, p. 152

${ }^{37}$ Vázquez, Luis Daniel y Serrano, Sandra. (2011) Los principios de universalidad, interdependencia, indivisibilidad y progresividad. Apuntes para su aplicación práctica en "La reforma constitucional de derechos humanos: un nuevo paradigma", Coords. Pedro Salazar Ugarte y Miguel Carbonell Sánchez, México, Instituto de Investigaciones Jurídicas, UNAM, p.152 y 153

${ }^{38}$ Vázquez, Luis Daniel y Serrano, Sandra. (2011) Los principios de universalidad, interdependencia, indivisibilidad y progresividad. Apuntes para su aplicación práctica en "La reforma constitucional de derechos humanos: un nuevo paradigma", Coords. Pedro Salazar Ugarte y Miguel Carbonell Sánchez, México, Instituto de Investigaciones Jurídicas, UNAM, p.159

${ }^{39} \mathrm{El}$ derecho a la salud reconocido como derecho fundamental se encuentra consagrado en gran cantidad de instrumentos jurídicos internacionales, los cuales fueron debidamente ratificados por México, en Bermúdez, Gabriela Mendizábal. "La atención a la salud en México." (2018), p.23 
afecciones o enfermedades" ${ }^{4041}$, mientras que el derecho humano a la salud es "el goce del grado máximo de salud que se pueda lograr..." ${ }^{42}$ El derecho humano a la salud ${ }^{43}$ se encuentra previsto en el artículo 4 párrafo cuarto de la Constitución Política de los Estados Unidos Mexicanos y su ley reglamentaria es la Ley General de Salud además de un cuerpo de reglamentos y normas específicas ${ }^{44}$. Cabe señalar que, al violentar el derecho a la salud, generalmente, hay un trato discriminatorio y omite el trato digno al paciente. La discriminación ${ }^{45}$ es la omisión del derecho y principio de igualdad jurídica $^{46}$. La ausencia de igualdad se constriñe a la inexistencia del trato igual entre iguales en mismas condiciones o circunstancias.

El Pleno de la Suprema Corte de Justicia de la Nación emitió una tesis donde explica claramente la diferencia entre discriminación directa e indirecta. La tesis lleva por rubro DISCRIMINACIÓN POR OBJETO Y POR RESULTADO. SU DIFERENCIA ${ }^{47}$. En esta tesis se explica que la discriminación ocurre cuando las normas y prácticas invocan explícitamente un factor prohibido de discriminación, a lo que le llama

${ }^{40}$ Ley General de Salud, artículo 1 bis

${ }^{41}$ OMS. Constitución de la Organización Mundial de la Salud. Formato PDF. Disponible en: https://www.who.int/governance/eb/constitution/es/

42 Ibidem

${ }^{43}$ Para hacer efectivo ese derecho humano se instrumentó el Sistema Nacional de Salud en los términos del artículo 5 de la Ley General de Salud

${ }^{44}$ La Ley reglamentaria que emanó de este artículo 4 párrafo cuarto constitucional es la Ley General de Salud y de ahí se derivó una serie de reglamentos, normas oficiales y guías de práctica médica.

${ }^{45}$ La discriminación tiene la intención de nulificar o menoscabar los derechos y las libertades de las personas, se considera un delito tanto en materia federal como en el estado de México.

${ }^{46}$ Principio de igualdad jurídica: trato igual a los iguales en las mismas circunstancias y condiciones.

${ }^{47}$ Gaceta del Seminario Judicial de la Federación. Tesis aislada. Décima época. Registro 2012597. Pleno. Libro 34, septiembre 2016. Tomo I. Materia Constitucional. Tesis P.VII/2016 (10 $\left.{ }^{\mathrm{a}}\right)$, p. 255 
"categoría sospechosa". La discriminación puede aparecer como causa motivadora de la distinción, exclusión, restricción o preferencia arbitraria e injusta, y es a lo que se le llama discriminación por objeto o discriminación directa. Sin embargo, la discriminación por resultado o discriminación indirecta puede ocurrir cuando las normas y prácticas son aparentemente neutras, pero el resultado de su contenido o aplicación constituye un impacto desproporcionado en personas o grupos en situación de desventaja histórica justo debido a esa desventaja, sin que exista para ello una justificación objetiva y razonable. ${ }^{48}$

Determinándose que la diferencia entre la discriminación directa de la indirecta radica en que la primera se encuentra regulada expresamente en la norma hacia un grupo en situación de vulnerabilidad, mientras que la indirecta puede aparecer también, cuando los efectos de su aplicación les generan un daño de discriminación a determinado grupo de personas. "Esto significa que una ley que, en principio, parezca neutra, podría tener efectos discriminatorios para cierto grupo de personas. " ${ }^{49}$ En definitiva, la discriminación es una práctica cotidiana en la atención obstétrica tanto en hospitales públicos como privados del país. Tratándose de mujeres embarazadas hay que considerar que existe el binomio materno - infantil lo que conlleva a considerar más derechos transgredidos. En este último caso estamos hablando del interés superior del menor ${ }^{50} \mathrm{y}$

${ }^{48}$ Gaceta del Seminario Judicial de la Federación. Tesis aislada. Décima época. Registro 2012597. Pleno. Libro 34, septiembre 2016. Tomo I. Materia Constitucional. Tesis P.VII/2016 (10 ${ }^{\mathrm{a}}$.), p.255

${ }^{49}$ Gaceta del Seminario Judicial de la Federación. Tesis aislada. Décima época. Registro 2012597. Pleno. Libro 34, septiembre 2016. Tomo I. Materia Constitucional. Tesis P.VII/2016 (10a.), p.255

${ }^{50}$ Regulado en el artículo 4 constitucional en su párrafo noveno y siguientes donde se precisa que el principio del interés superior de la niñez son acciones encaminadas a garantizar el desarrollo pleno de los menores para que gocen de una vida digna, tanto en condiciones materiales como afectivas que les permita vivir con completa satisfacción y bienestar posible. 
del derecho a la identidad ${ }^{51}$ en el caso de que fuera rechazada la atención médica por parte del centro de salud. En otros casos más delicados, en donde se pone en peligro la vida, seguridad y salud de las personas, se puede considerar vulnerado el derecho a la integridad y seguridad personal ${ }^{52}$, incluso en caso de muerte, el derecho a la vida.

También existe la violencia psicológica ${ }^{53}$, la que se define como el trato deshumanizado ${ }^{54}$ por parte del personal médico. Este considera tres tipos de prácticas: 1) la omisión de la información, 2) el trato deshumanizado, y 3 ) actos u omisiones que impliquen discriminación o humillación. ${ }^{55} \mathrm{Al}$ concretarse cualquiera de esas conductas se configura el mal trato con lo que se atenta contra la dignidad humana ${ }^{56}$, pero también en discriminación de sus derechos. Lo delicado de las prácticas de discriminación - que a la vez generan

${ }^{51}$ También regulado en el artículo 4 párrafo octavo donde se le reconoce el derecho a la identidad y a ser registrado de manera inmediata a la persona recién nacida garantizándole el Estado con ello sus derechos.

${ }^{52} \mathrm{Al}$ privarle de sus derechos a un individuo se está atentando contra el pleno desarrollo de la persona, conocido como derecho a la integridad. El derecho a la integridad personal implica la prohibición a la pena de muerte, a la tortura y de penas o tratos crueles, inhumanos o degradantes, consagrados en el artículo 22 constitucional.

${ }^{53}$ La violencia psicológica esta considerada como un delito previsto en el artículo 282 del Código Penal del Estado de México.

${ }^{54}$ Ley General de Acceso a las Mujeres a una Vida Libre de Violencia, artículo 6, fracción I

${ }^{55}$ Comisión Ejecutiva de Atención a Víctimas. (CEAV) (2017) "Diagnóstico sobre victimización a causa de violencia obstétrica en México.” [En línea] Disponible en: https://www.gob.mx/cms/uploads/attachment/file/194701/Diagno_stico_VO_port. pdf, [Consultado 8 de febrero de 2019] p.6

${ }^{56}$ En la tesis aislada P. LXV/2009 publicada en el Semanario Judicial de la Federación y su Gaceta, Tomo XXX, Diciembre de 2009, página 8, la SCJN reconoce el valor superior de la dignidad humana, en cuanto a que ésta es necesaria para que los individuos desarrollen integralmente su personalidad, puesto que se consideran implícitos otros derechos como el derecho a la vida, a la integridad física y psíquica, al honor, a la privacidad, al nombre, a la propia imagen, al libre desarrollo de la personalidad, al estado civil y el propio derecho a la dignidad personal. 
violencia hacia las personas - es que han decantado en la creación de un nuevo tipo punitivo ${ }^{57}$. Éste considera que se atenta contra la dignidad humana, por tanto, el resultado perjudicial que prohíbe el derecho penal es "la intención de nulificar o menoscabar los derechos y las libertades de las personas". ${ }^{58}$

Es notorio que los principios de interdependencia e indivisibilidad que señala la teoría de los derechos humanos están presentes, por ello la observancia de los derechos humanos debe ser una práctica obligada por todas las personas y enfatizarse en los servicios de salud.

Esta misma teoría señala que el garante de los derechos humanos es el Estado y para ello cuenta con obligaciones y deberes. Para el caso de México esas obligaciones y deberes se encuentran plasmados en el artículo $1^{\circ}$, tercer párrafo de la Constitución Política de los Estados Unidos Mexicanos. Por un lado, se encuentran las obligaciones de promover, respetar, proteger y garantizar los derechos humanos ${ }^{59}$ de conformidad con los principios rectores ya estudiados, y por otro lado con el deber de prevenir, investigar, sancionar y reparar las violaciones a los derechos humanos ${ }^{60}$. Ante toda la problemática que presenta el Sistema Nacional de Salud, el Estado Mexicano es el responsable de proteger y garantizar el derecho humano a la salud y de sancionar y reparar las violaciones al mismo. ${ }^{61}$

${ }^{57}$ Código Penal para el Distrito Federal (hoy Ciudad de México), artículo 206 fracción III

${ }^{58}$ Gaceta del Semanario Judicial de la Federación. Tesis: Aislada. Décima Época. Registro: 2005489. Sexto Tribunal Colegiado En Materia Penal Del Primer Circuito. Libro 3, febrero de 2014, Tomo III. Tesis: I.6o.P.42 P (10a.), p.2310

${ }^{59}$ Carbonell, Miguel. Teoría de los Derechos humanos y del control de convencionalidad, Instituto de Investigaciones Jurídicas, UNAM, quinta edición, México, 2014, p.18

${ }^{60}$ Becerra Ramírez, Manuel, Artículo 10., tercer párrafo. Prevenir, investigar, sancionar y reparar como deberes del Estado frente a violaciones de derechos humanos, en "Derechos humanos en la Constitución. Comentarios de jurisprudencia constitucional e interamericana, t. I", Cords. Ferrer Mac-Gregor, Eduardo, Caballero Ocha, José Luis y Steiner, Christian IIJ-UNAM, SCJN, Fundación Konrag Adenauer, México. 2013, p.140

${ }^{61}$ Salazar Ugarte, Pedro y otros, Las obligaciones del Estado, en "La reforma 
Lo relevante es que, tratándose de centros de salud pública, sin importar quién sea el servidor público que cometa la infracción, de acuerdo con la legislación, ésta es considerada una Responsabilidad del Estado Mexicano ${ }^{62}$.

\section{ili. Metodología}

Para la investigación de campo se utilizó la metodología ex post facto $^{63}$. Se elige este tipo de metodología por considerarse la más apropiada para establecer posibles relaciones de causa-efecto. La metodología ex post facto: ... es un planteamiento contrario al normalmente usado. Aquí sucede que el investigador, a diferencia de lo que ocurre en la metodología convencional, no puede manipular la variable independiente, porque ésta ya se presentó y produjo sus efectos sin que el investigador los conozca; pero sí está en condiciones de analizar el efecto, es decir la variable dependiente, para tratar de identificar las causas que lo produjeron. ${ }^{64}$

En el campo médico, desde la postura jurídica, se analizan los efectos de determinados actos en la salud humana que han producido trasgresión a derechos humanos en las personas, buscándose las causas que los han ocasionado para minimizar sus efectos.

Para el desarrollo de la técnica de campo se determinaron lineamientos metodológicos ${ }^{65}$ que sirvieron para establecer la línea

constitucional sobre derechos humanos. Una guía conceptual", Instituto Belisario Domínguez, Senado de la República, Ciudad de México, 2014, p.119

${ }^{62}$ Salazar Ugarte, Pedro y otros, Las obligaciones del Estado, en "La reforma constitucional sobre derechos humanos. Una guía conceptual”, Instituto Belisario Domínguez, Senado de la República, Ciudad de México, 2014, p.121

${ }^{63}$ ex post facto, su significado después que sucedieron los hechos. Esta metodología es muy común que se ocupe en el campo del derecho, ya que se requiere que se produzca el efecto jurídico para que se inicie su estudio.

${ }^{64}$ Mejía Mejía, Elías, Metodología de la investigación científica, Lima, Universidad Nacional Mayor de San Marcos, 2005, p. 186

${ }^{65}$ Ponce de León Armenta, Metodología del derecho, México, Porrúa, 2011, p. 130. 
de investigación. Se precisó como objetivo de estudio: Analizar los sucesos que originan la trasgresión de los derechos humanos en el Hospital Escuela del IMSS, ubicado en el estado de México, con el fin de identificar las causas de las incidencias y cómo interactúan los alumnos de medicina en el Sector Salud. Se eligió esta institución de salud, ya que es un hospital general de segundo nivel, que alberga la mayor parte de las especialidades médicas. Además, es un hospital-escuela con diversas categorías de alumnos que constituyen el universo ${ }^{66}$ de estudio de esta investigación.

El universo determinado para la aplicación de la técnica de campo lo conforman los profesionales de la salud, alumnos universitarios, de pregrado y posgrado. Esta fase se apoyó en opiniones de médicos especialistas, médicos profesores y autoridades del mismo hospital.

Se trazó como hipótesis ${ }^{67}$ principal: Los profesionales de la salud omiten pasos en los procedimientos, ocasionando involuntariamente error médico y trasgresión a los derechos humanos de las pacientes en el Hospital; y como hipótesis secundaria ${ }^{68}$ : La educación en derechos humanos en el campo medico sensibilizará a los alumnos universitarios, de pregrado y posgrado para brindar una atención médica con trato humanizado y evitar responsabilidad profesional. Básicamente, para la investigación ex post facto es imposible tener control sobre las variables independientes, puesto se deben tomar las cosas tal como son e intentar descifrarlas a través de las variables dependientes. Por otro lado, se propuso contar con un grupo de control dividido en tres categorías: alumnos universitarios, internos

${ }^{66}$ Se denomina Universo a la población sobre la que versará el estudio.

${ }^{67}$ Se puede definir a la hipótesis como la respuesta tentativa a un problema objeto de investigación, tal y como señala Muñoz Rocha, Carlos en Metodología de la Investigación, Oxford, México, 2015, p.196

${ }^{68}$ Para este trabajo se utilizaron hipótesis de investigación, que con base en la metodología ex post facto se requiere vincular a estas con las variables, por ello se eligió una hipótesis principal y una secundaria. 
de pregrado y residentes de posgrado. ${ }^{69}$

\section{EVIDENCIAS EMPÍRICAS}

En esta etapa de la investigación, se tomó como muestras ${ }^{70}$ experimentales situaciones reales que se produjeron espontáneamente en los actos médicos. Se obtuvo una explicación entre las relaciones causales y los elementos: la práctica médica y la violación a los derechos humanos. La intención fue indagar sobre los procesos que siguen los jóvenes médicos y demás profesionales de la salud y relacionarlos con los derechos humanos para visibilizar la manera en que interactúan los estudiantes en ese ámbito. Esta parte se complementó con las técnicas de la entrevista no estructurada, la observación directa y no estructurada, el diario y libreta de notas. ${ }^{71}$

Se diseño el taller de sensibilización en materia de derechos humanos $^{72}$ que se impartió al grupo de control dividido en las categorías: alumnos universitarios, internos de pregrado y residentes de posgrado. El taller para alumnos universitarios de los semestres quinto, séptimo y octavo se celebró en dos sesiones de una hora y media los miércoles 21 y jueves y 22 de agosto de 2019. El taller para alumnos internos de pregrado se realizó en tres bloques de una hora los miércoles 25, jueves 26 y viernes 27 de septiembre de 2019 y, por último, el taller para alumnos de posgrado fue celebrado el 26

${ }^{69}$ Este grupo de control en sus tres categorías fueron alumnos seleccionados por el jefe de enseñanza del Hospital sin que se conociera su identidad, ni ningún dato que los pudiera individualizar previamente a las sesiones programadas con ellos.

${ }^{70}$ La muestra es el segmento de la población que se considera representativa de un universo y se selecciona para obtener información acerca de las variables objeto de estudio, en Muñoz Rocha, Carlos, Metodología de la Investigación, Oxford, México, 2015, p. 220

${ }^{71}$ Todos estos elementos forman parte de la técnica de investigación empírica.

${ }^{72}$ Este Taller se derivó de los resultados obtenidos de la investigación documental, en donde se visibiliza el desconocimiento de los derechos humanos en el campo médico y que se requiere verificar con la investigación empírica. 
de febrero de 2020 durante la semana de capacitación e inducción que reciben los residentes, previo a la incorporación a sus servicios respectivos de la especialidad en el hospital. Fue una sesión única de dos horas y media.

\section{Entrevista con el Jefe de enseñanza}

Una semana antes, de iniciar el primer curso me presenté con el Jefe de Enseñanza, para organizar los grupos y me diera instrucciones para la impartición del taller. En esa entrevista me indicó que se había detectado que "las recomendaciones que llegan son para los médicos, pero también para el personal de enfermería y para las señoritas del área administrativa por dar mal trato a pacientes y familiares", o bien, el llamado trato deshumanizado en hospitales. Comentó que "este primer grupo era de alumnos de los últimos semestres de la universidad y que había algunos que empezarían el próximo año su internado en el hospital. Estos jóvenes nunca han tenido contacto con el tema de los derechos humanos, por lo cual el taller era muy conveniente”. Ese mismo día, el jefe de enseñanza me enseño el auditorio del hospital, lugar donde se impartió el curso. Es un recinto con capacidad para 100 personas, pero para el taller se estaba considerando un grupo de 80 alumnos, cuya asistencia era obligatoria. Me apoyo con parte de la impresión del material, pero no tuvieron acceso a los instrumentos de evaluación.

Categoría 1. Alumnos Universitarios de Medicina de la Facultad de Estudios Superiores Iztacala, UNAM.

El 22 de agosto de 2019, los alumnos en su totalidad de la UNAM tardaron en entrar al auditorio, los primeros ingresaron a las 11:05 horas y los últimos llegaron a las 11:45 horas. Éstos últimos fueron los alumnos de $8^{\circ}$ semestre, quienes ya mostraban actitud de superioridad ante los demás, ejerciendo las jerarquías dentro de la 
profesión $^{73}$. A estos últimos se les tuvo que pedir orden para que se condujeran apropiadamente dentro del recinto. El tema de la sesión fue el error médico, que conduce a la negligencia y a la violencia en hospitales. Se explicó la diferencia entre estos conceptos y cómo su ejecución produce delitos como el de responsabilidad profesional. Resulta interesante observar que los alumnos solicitaron un curso de derechos de los médicos para saber cómo se podían defender y ante quién podían acudir. Otra inquietud fue reconocerse incapaces de percibir cuándo realizan actos de discriminación a los pacientes. Se destaca que son los mismos médicos profesores con quienes realizan sus prácticas profesionales son quienes que giran esas instrucciones a los alumnos para que ignoren a los pacientes.

Por un período vacacional del Jefe de Enseñanza se pospusieron los talleres. En tanto eso sucedía, el 5 de septiembre de 2019 alrededor de mediodía me dirigí al hospital en comento para agradecer personalmente al director del hospital el que me haya permitido realizar esa primera actividad. En esa entrevista se utilizó la técnica de la entrevista no dirigida, ${ }^{74}$ porque sólo fue una charla ligera y sin formalismos. Fue amable y comentó que existen problemas relacionados con la violación a los derechos humanos entre casi todo el personal. Al parecer, la gente que acude con sus pacientes se queja directamente por el mal trato que reciben ante la Comisión Nacional de los Derechos Humanos o a la Comisión Estatal de Derechos Humanos. "El mayor problema es con el personal administrativo, que brinda una atención sin calidez humana", comentó. En cuanto a las quejas por los médicos, no aclaró si son por una responsabilidad institucional o profesional. Al dirigir la conversación, me permití ofrecer algunas sugerencias: le comenté que se debería tener

${ }^{73}$ En esta primera sesión dejo ver que los estudiantes de octavo semestre ejercen el curriculum oculto que es parte de la formación como médicos, como se refirió en el habitus médico.

${ }^{74}$ Ibáñez, Alejandro Acevedo, and Alba Florencia A. López Martín. El proceso de la entrevista: conceptos y modelos. Editorial Limusa, 2004, p.29 
mucho cuidado con el asunto de la violencia obstétrica, ya que ahí es donde están los delitos previstos en el código penal del estado de México. Respondió “ya los hemos experimentado y ya hemos trabajado al respecto. Esa área la tenemos casi controlada para no incurrir en delitos. Sin embargo, se siguen vulnerando derechos humanos". Me comentó "en Oncología se ha desatado la violencia de médicos y residentes hacia los pacientes y familiares de los pacientes. Son acontecimientos muy difíciles de controlar y prever”, me dijo. Y eso nos llevó al tema de los alumnos de posgrado que cursan su especialidad, cuya edad promedio esta alrededor de los 30 años. ${ }^{75}$ "Ellos son los más rebeldes y difíciles de tratar. Exigen sus derechos y casi no quieren estudiar con el ahínco con que lo hacían otras generaciones”. Me explicó que por esa dedicación de servicio los médicos eran respetados y reconocidos por toda la población, pero ahora "las nuevas generaciones quieren trabajar poco, retirarse a sus horas oficiales de salida sin la menor inquietud". También aseveró que "la medicina se aprende con la práctica, no tanto en el aula universitaria, sino en la clínica, practicando". Habló de la violencia en la sociedad y de cómo ha permeado en el hospital. De esa conversación destacó que la capacidad hospitalaria está rebasada por 200 mil pacientes, ya que ésta es de hasta 800 mil personas, pero a la fecha (2019) se encontraban atendiendo a un millón de personas. Me expresó su agradecimiento por estar impartiendo esos talleres y me invitó a continuar por el tiempo que yo quisiera, lo cual agradecí y aproveché para ofrecer la capacitación a los médicos titulados del hospital, con un taller enfocado en derechos humanos, pero dirigido a la responsabilidad profesional. Al parecer, el personal del hospital se comunicó entre sí y señalaron que estaba un representante de Derechos Humanos, pero nunca aclararon que

${ }^{75}$ La generación que actualmente cuenta con una edad de 30 años pertenece a la población por estadística demográfica conocida como Millennials, en Ramírez Arias, José Luis, Federico Rodríguez Weber, and Raquel Ocampo Lujano. "La educación médica para las últimas generaciones." Acta médica Grupo Ángeles 16.3 (2018): 267-270. 
yo era una académica y que sólo iba a impartir los talleres de capacitación en la materia, por lo que poco a poco personal del hospital, representantes del sindicato, representantes de enfermería, entre otros se fueron presentando en la oficina, para escuchar lo que se hablaba. Ante ello, el director fue más parco en sus respuestas y yo entendí que la entrevista había concluido, de modo que me despedí y salí de su oficina.

\section{Categoría 2. Estudiantes internos de pregrado}

Día 1. El 25 de septiembre de 2019, a las 13:30 horas me presenté acompañada por una observadora, quien atendió el control de asistencia y la aplicación de los instrumentos de medición. El jefe de enseñanza me proporcionó el equipo de sonido y de cómputo para realizar el taller. Su técnico auxiliar nos ayudó en la instalación del equipo y sonido, ya que en esta ocasión se presentó un vídeo. A las 14:08 horas ya había alrededor de 30 estudiantes, por lo que el curso dio inicio. La observadora me ayudó a repartir el material y al control de asistencia. Los estudiantes tenían entre 22 y 26 años. Expresaron que en su grupo sólo había cinco estudiantes provenientes del Instituto Politécnico Nacional (IPN) y el resto eran de la FES Iztacala (UNAM). Este grupo fue más participativo. Sobre todo, cuando se explicaron los derechos humanos que tienen injerencia en el Sector Salud. Las preguntas e inquietudes empezaron a manifestarse, principalmente con el tema de violencia: cómo evitar la violencia de los pacientes y familiares ejercida en contra de los médicos. Planteando sus experiencias y lo que habían hecho para resolverlo o agravarlo, en su caso. Después de dejarlos que interactuaran, se les dio la opinión jurídica para evitar entrar en conflictos legales. Otro asunto que llamó su atención fue el consentimiento informado en la sala de urgencias. Este es el derecho a la información que se traduce a la información clara y oportuna sin dejar lugar a dudas sobre el padecimiento y el procedimiento que se le va 
a realizar al paciente. Con estos dos temas se realizaron los grupos de discusión, donde se expresaron las inquietudes y se resolvieron dudas sobre la actuación de los jóvenes médicos y cómo evitar ser objeto de violencia y de responsabilidad profesional. Con esta dinámica concluyó la primera sesión.

Día 2. El 26 de septiembre de 2019 se presentó un vídeo ${ }^{76}$ donde se mostró como una persona asistente médico negó otorgar el servicio de atención médica y de medicamentos en un hospital de IMSS ubicado en La Quebrada, estado de México. La intención era que reconocieran los derechos humanos que se vulneraban en dicha circunstancia. Es relevante mencionar que no todos los estudiantes eran los mismos que en la primera sesión. En esta ocasión se trabajó en dinámica grupal la solución de un caso práctico de los derechos reproductivos de una mujer menor de edad. El 90\% de los internos comentaron que no le aplicarían un anticonceptivo porque no querían caer en un supuesto de responsabilidad profesional. Sólo una minoría decidió apoyar a la joven con un método anticonceptivo apropiado a las necesidades de su cuerpo, sin que existieran consecuencias jurídicas para ninguna de las partes. Acto seguido se explicó lo que era el error médico, cómo se construía y las consecuencias que derivan de él. También se indicó lo que debía entenderse por violencia en el ámbito de la salud y cómo se convertía en cocreadora de algunos delitos que impactaban directamente en la práctica médica. A lo que indicaron como ellos habían respondido en esas circunstancias, algunos con trato arbitrario.

Incidencia. Al concluir la sesión un alumno se acercó para preguntarme si él tenía alguna responsabilidad porque, durante su servicio en piso, la familiar de un paciente, sin importarle las reglas del hospital, aun cuando ella también es trabajadora del IMSS de otro nosocomio, había tomado el carrito de curaciones, y le había rea-

\footnotetext{
${ }^{76}$ Video grabado por la paciente y difundido en redes. "Empleada del IMSS niega insulina a paciente". AM noticias. Disponible en: https://www.youtube.com/watch?v=J66SczShp_U
} 
lizado la curación al paciente. Cuando llegó el interno, a quien le correspondía esa actividad, notó lo sucedido y le dijo a la familiar: "Usted no puede hacer esto". Ante ello, la mujer se tornó agresiva y le contestó casi a gritos "no le han hecho las curaciones a mi paciente y no le han dado informes médicos de la situación”. El interno abrumado y molesto se retiró sin confrontarla, pero lo asentó en el expediente clínico. No supo qué sustancia o medicamento le aplicó, ni qué procedimiento siguió. Le avisó a la jefa de enfermeras y ésta última tomó cartas en el asunto. Acto seguido, la enfermera le dijo al interno que se pusieran de acuerdo para que coincidieran las notas médicas en el expediente clínico ${ }^{77}$. La mayor preocupación del interno era su situación jurídica. Se le explicó que él no era responsable mientras fuera estudiante, pero el médico tratante sí lo era. Así que debería avisarle, pero comentó que el médico tratante se había retirado. Sin embargo, avisó a los de la siguiente guardia. Quedó tranquilo cuando se le mencionó que había procedido correctamente.

En la última sesión se planeó una estrategia para tomar la asistencia y reconocer a los internos que se anotaban en la lista, pues ellos agregaban a otros compañeros. La observadora estuvo al pendiente de quiénes firmaban y aproximadamente cinco estudiantes pretextaron que debían ir a entregar papeles, más nunca regresaron $^{78}$. Al iniciar el curso se empezó dando un repaso de lo que es el error médico para dar entrada al tema de los delitos. En esta ocasión expresaron muchas inquietudes sobre la violencia de la que son objeto por parte de los pacientes y sus familiares. Al finalizar el jefe de enseñanza abordó las problemáticas que se estaban presentando: el tema de violencia que sufrió su compañero interno, el acoso sexual que están sufriendo algunas alumnas en el hospital y

${ }^{77}$ Estas acciones visibilizan como el personal de salud más experimentado, instruye a los alumnos de medicina a manejar las notas medicas que integran el expediente clínico.

${ }^{78}$ Fue notorio el desinterés en el curso y las prácticas deshonestas con la alteración y la falsificación de firmas de los compañeros ausentes. 
los robos entre los internos. Les señaló la importancia de cumplir con el reglamento y sobre la limpieza de sus uniformes, así como de los lugares donde descansan por las noches. Mencionó que por el incumplimiento del reglamento en las faltas debían cubrir las inasistencias durante sus vacaciones. De esta charla se muestra que el habitus médico persiste, aun cuando ha cambiado en el sentido de que los profesionales de la salud ahora están más protegidos y se les impide tomar decisiones por sí mismos. Además de que el servicio social ya lo realizan en las ciudades por la inseguridad que existe en el país. Las medidas disciplinarias siguen existiendo, sobre todo porque los alumnos han quebrantado una regla de conducta.

El 8 de noviembre de 2019 me presenté en el hospital y me enteré qué el jefe de enseñanza se había jubilado. En ese momento, se me presentó al nuevo jefe, con quien platiqué un poco y me pidió tiempo en lo que tomaba posesión del puesto. Me indicó que lo buscara en enero de 2020.

Categoría 3. Estudiantes residentes de posgrado

El 14 de enero de 2020, el nuevo Jefe de Enseñanza, me informó que tenía programada la fecha para el curso de los residentes para el 26 de febrero del 2020, sería durante la semana de capacitación e inducción que reciben, previo a la incorporación a sus servicios de la especialidad en el hospital. Sería una sesión única de dos horas y media. El día del curso inicié a las 11:20 horas y noté que eran aproximadamente 42 alumnos los que ocupaban el auditorio. Todos médicos titulados iniciando su primer año de residencia. Su edad fluctuaba entre los 24 y los 30 años. Después de presentarme y expresar los agradecimientos, al director del Hospital y al jefe de enseñanza, inicié la exposición. Los temas les resultaron de interés a medida que se avanzaba y sobre todo cuando empezaron a intervenir con el caso práctico. Se pudo notar que tenían diferentes criterios para identificar un menor de edad y el médico que debería 
tratarlo. Solo los médicos residentes con especialidad en pediatría sabían la respuesta correcta. Posteriormente al llegar a la respuesta del caso práctico, surgieron más interrogantes. Se destaca que ellos siguen las guías elaboradas por la secretaría de salud, las cuales dan imprecisiones legales al tratarse de menores de edad, lo cual fue notorio cuando se hizo la discusión. "La guía dice que los menores de edad si pueden decidir sobre su cuerpo", aludió una alumna. Creció la discusión. Finalmente, les compartí la respuesta legal con el procedimiento y los requisitos que ha señalado la Suprema Corte de Justicia de la Nación. Se hicieron comentarios generales, pero finalmente un alumno infirió, "sí no quiero tener problemas legales, aunque la guía diga que se puede, mejor no lo hago". En ese sentido asintieron muchos de ellos. Lo que más les llamó la atención fueron las sanciones a las que pueden ser acreedores si no siguen las reglas previstas por las Normas Oficiales Mexicanas ya que existía desconocimiento de su existencia. Este grupo fue más sigiloso en sus experiencias y conductas médicas, tampoco sabían sobre el error médico y como se integra. Solo reconocieron conocer la negligencia médica, pero ya con experiencia de como evitarla en el expediente clínico.

\section{v. Hallazgos}

Después de haber procesado la información y analizado los datos se obtuvieron los siguientes hallazgos.

Hallazgo 1. Los estudiantes universitarios de medicina desean aprender sobre derechos humanos y responsabilidad profesional médica

Estudiantes de los semestres quinto, séptimo y octavo de medicina. De un universo de 80 alumnos que integró este grupo con edades que fluctuaban entre los 20 y 22 años, solo se pudo aplicar el instrumento de medición a 62 alumnos, por lo que con este número se tomó la muestra. Por el rango de edades, cabe advertir que este 
grupo está dentro de la generación demográfica llamada Centennials. En función a los alumnos con posibilidad de vulnerar derechos humanos, los datos obtenidos muestran un riesgo alto de 28 alumnos lo que equivale a $35 \%$; un riesgo medio de 24 alumnos lo que equivale a $30 \%$, y un riesgo bajo con 28 alumnos lo que equivale a 35\%. En total hubo un aprovechamiento de: $84 \%$ con la calificación más alta de 8.8 obtenida por 4 alumnos de séptimo semestre.

Cabe señalar que este grupo se mostró preocupado por el error médico y saber más sobre la responsabilidad médica. Se destaca que éste también manifestó estar interesado en repetir el curso y a que se les impartiera un curso sobre los derechos de los médicos.

Hallazgo 2. Los Estudiantes De Pregrado Y Posgrado Son Más Propensos A Vulnerar Derechos Humanos

Alumnos de pregrado - internos. De un universo de 56 alumnos que integró este grupo solo se consideró una muestra de 26 alumnos para el instrumento de medición. Las edades fluctuaban entre 22 y 26 años, lo que hace que este grupo se considere dentro del margen de dos generaciones demográficas millennials y centennialls puesto que este grupo estaba conformado por la transición entre una y otra. En función a los alumnos con posibilidad de vulnerar derechos humanos los datos obtenidos muestran un riesgo alto de 34 alumnos lo que equivale a $61 \%$. En este rubro se consideraron a los ausentes que inasistieron a la evaluación. Un riesgo medio a 10 alumnos el cual equivale a $21 \%$ y un riesgo bajo de 12 alumnos que equivale a $21 \%$. El aprovechamiento fue de $84 \%$, pero en función a la muestra de 26 alumnos ya que el universo era de 56 alumnos por lo que este dato no es definitivo. La calificación más alta fue de 9.2 y la obtuvo una alumna mujer.

Se subraya que este grupo mostró demasiadas conductas asociadas a la falta de ética y valores. Mintieron con mucha facilidad para evitar tomar el curso, falsificaron firmas - un total de 46 falsificaciones - y presunción de asumir otra identidad, todas ellas son consideradas falta de probidad. A este grupo se le recomendó trabajar 
con la ética médica. En el informe que se presentará al director del hospital se sugiere repetir el curso ya que el riesgo de vulnerar derechos humanos y cometer delitos es alto. Aun cuando este grupo no mostró mayor interés de repetir el curso ni conocer sobre derechos humanos. Su mayor inquietud fue resolver sobre la violencia física y de acoso sexual que sufren en su persona.

Hallazgo 3. Los residentes evitan caer en problemas legales

Alumnos de Posgrado - Residencia Médica de primer año. De un universo de 40 alumnos de posgrado que integro este grupo se consideran 37 residentes de primer año y 3 de servicio social. En este curso no hubo ausentismo porque se realizó en una sola sesión y todos permanecieron de inicio a fin en el recinto. Las edades fluctuaban entre 24 y 30 años, correspondiéndoles la generación demográfica Millennials. En función a los alumnos con posibilidad de vulnerar derechos humanos los datos obtenidos muestran un riesgo alto de 8 estudiantes lo que equivale a 20\%, un riesgo medio de 14 estudiantes lo cual equivale a 35\% y un riesgo bajo de 18 estudiantes que equivale a $45 \%$. El aprovechamiento fue de $87 \%$, aun cuando se hace notar que la calificación más alta fue de 8.4, correspondiéndoles a 3 alumnos de residencia médica y uno del servicio social. Se destaca que aun cuando el aprovechamiento fue mayor la calificación más alta es menor que la que obtuvieron los alumnos de pregrado y estudiantes universitarios.

Recomendación trabajar con la ética médica. Se sugiere repetir el curso ya que son médicos titulados con experiencia profesional, a quienes se les han muerto pacientes y conocen los riesgos de la responsabilidad profesional médica, por lo que evitan caer en un problema legal. Además, señalaron desconocer los derechos humanos en la práctica médica y sus implicaciones. 


\section{vi. Conclusiones}

La investigación arrojó que los profesionales de la salud tienen desconocimiento sobre derechos humanos y la responsabilidad jurídica a la que son acreedores por una conducta contraria a Derecho. Además, el Taller de Sensibilización de Derechos Humanos impartido a alumnos universitarios, de pregrado y posgrado en hospitales-escuelas de instituciones públicas de salud reveló que los estudiantes de medicina de la UNAM no cursaron la asignatura ética médica, lo que constituye un factor que durante su estancia en éste vulneren los derechos humanos de los pacientes.

Ante la violencia que existe en la sociedad y que ha permeado en los hospitales, los estudiantes de medicina desean conocer sus derechos y las implicaciones legales de sus conductas causadas por error médico, pues existen quejas por negligencia. El error médico que se presenta en hospitales públicos es, generalmente, inexcusable lo que conlleva que se finque una responsabilidad administrativa al médico tratante y un acta administrativa a los estudiantes. En cuanto a la apreciación de los derechos humanos, los profesionales de la salud mostraron gran interés por la capacitación en materia de derechos humanos y en ampliar su cultura de legalidad para conocer las responsabilidades médicas, sus derechos y cómo ejercerlos.

La problemática que presenta el sistema de salud pública en el país se ha agudizado en el actual gobierno por el presupuesto reducido que se presentó para el período 2019-2024 y la aparición del coronavirus SARS-GoV-2, lo cual vino a evidenciar las grandes carencias hospitalarias en infraestructura, malas condiciones laborales del personal de la salud y las prácticas de corrupción que aún prevalecen, por lo que el incumplimiento de las obligaciones del Estado mexicano en el ámbito de sus competencias en materia de salud, se visibilizan como una actividad administrativa irregular, ocasionando violencia institucional dirigida al personal de salud que repercute a pacientes con trato arbitrario y deshumanizado. Lo que todo apunta a un estudio de responsabilidad del Estado en el sector salud. 
En el Hospital del IMSS, donde se realizó la investigación empírica, - a partir de marzo del 2020 es dedicado a pacientes Covid-19-, aunque ha disminuido la violencia obstétrica dentro de sus instalaciones, siguen prevaleciendo las trasgresiones a los derechos humanos, por ello es obligada la capacitación por lo menos una vez al año, donde se profundice en aspectos de derechos de los pacientes y de los médicos. Además, se debe reforzar la ética médica a todo el personal de la salud y resolver inquietudes sobre la violencia ejercida sobre ellos.

No se puede dejar de observar que el 8 de mayo de 2020 se publicó la reforma en donde se inserta en el artículo 4 párrafo cuarto constitucional "un sistema de salud para el bienestar para la atención integral y gratuita de las personas que no cuenten con seguridad social" y una reforma en cascada a la Ley General de Salud, lo que provocaría un cambio de paradigma. Sin embargo, el COVID, vino a replantear el funcionamiento y organización del sistema nacional de salud y por el momento solo hay incertidumbre.

En suma, se corrobora a) el habitus médico se está modificando y sigue en transformación; b) la violencia institucional provoca conductas deshumanizadas y c) los médicos de edades entre los treintas que pertenecen a la generación demográfica Millennials son en los que recaen la mayor parte de negligencias médicas. El riesgo de vulnerar derechos humanos y cometer delitos en hospitales por parte del personal de la salud es alto, por ello es urgente diseñar un modelo jurídico basado en la educación en derechos humanos y cultura de la legalidad aplicable tanto al Sector Salud como al del Derecho donde se integren estrategias para coadyuvar en mejoras a la educación con el fin de armonizar la interdisciplina de las ciencias médica y del derecho. No obstante, se deberá esperar a que la epidemia del coronavirus SARS-CoV-2 (Covid-19) encuentre una vacuna cierta que aminore esta epidemia para estar en condiciones de implantarse. 
R6VISTA DEL POSGRADO EN DERECHO DE LA UNAM | revistaderecho.posgrado.unam.mx AÑO 8, No 15, JULIO - DiCIEMBRE 2021 | https://doi.org/10.22201/ppd.26831783e.2021.15.186

\section{Fuentes}

Bañuelos, Claudio, "Investigan muerte de madre y su bebe por negligencia en el ISSSTE”, en La Jornada, México, ciudad de México, 15 de noviembre de 2019. Disponible en: https:// www.jornada.com.mx/ultimas/sociedad/2019/11/15/pornegligencia-muere-madre-y-su-bebe-en-el-issste-de-aguascalientes-8551.html

Becerra-Ramírez, Manuel, "Artículo 1o., tercer párrafo. Prevenir, investigar, sancionar y reparar como deberes del Estado frente a violaciones de derechos humanos", en Eduardo Ferrer Mac-Gregor, et al. (coord), Derechos humanos en la Constitución. Comentarios de jurisprudencia constitucional e interamericana, t. I, México, IIJ-UNAM-SGJN-Fundación Konrag Adenauer, 2013.

Benassini, Oscar. "Atención en salud mental regionalización y reorientación en el marco de la descentralización de servicios de salud en México. "Salud Mental 20.4 (1997): 48-53.

Bermúdez, Gabriela Mendizábal, La atención a la salud en México. Morelos, Fontamara, 2018.

Carbonell, Miguel. Teoría de los Derechos humanos y del control de convencionalidad, México, Instituto de Investigaciones Jurídicas, UNAM, quinta edición, 2014.

Castro, Roberto y Joaquina Erviti, Sociología de la práctica médica autoritaria. Violencia obstétrica, anticoncepción inducida y derechos reproductivos, Cuernavaca, UNAM, 2015.

Castro-Pérez, Roberto, "Génesis y práctica del habitus médico autoritario en México". Revista Mexicana de Sociología 76, núm. 2, abril-junio, 2014

CEAV. "Diagnóstico sobre victimización a causa de violencia obstétrica en México", Comisión Ejecutiva de Atención a Víctimas. 2017, Disponible en: https://www.gob.mx/cms/uploads/ attachment/file/194701/Diagno_stico_VO_port.pdf 
CEDAW. Convención sobre la eliminación de todas las formas de discriminación contra la mujer. Disponible en: https://www. ohchr.org/SP/ProfessionalInterest/Pages/CEDAW.aspx

Código Penal del estado de México

Código Penal Federal

Código Penal para el Distrito Federal (hoy Ciudad de México)

Constitución Política de Los Estados Unidos Mexicanos

Declaración Universal de los derechos humanos.

Dreser, Anahí, et al. "Uso de antibióticos en México: revisión de problemas y políticas.” Salud pública de México, 50 (2008): S480-S487.

EDitor MF, "Se recortó presupuesto al sector Salud en pandemia", en Momento Financiero, México, ciudad de México, 22 de julio de 2020, Disponible en: https://momentofinanciero.mx/ recorte-salud/

EDitor. "Con camioneta, derriban portón para rematar a hombre en hospital de Guanajuato", en El Universal, México, ciudad de México, 9 de junio de 2018, Disponible en: https://www. eluniversal.com.mx/estados/con-camioneta-derriban-porton-para-rematar-hombre-en-hospital-de-guanajuato

GIRE. "Capítulo 4. Violencia Obstétrica", en Informe Omisión e Indiferencia. Derechos Reproductivos en México, Grupo de Información en Reproducción Elegida, AC, México, 2013.

Hernández, Alfonso. La teoría jurídica de los derechos humanos; el orden que surge del caos. Perspectiva desde el trabajo de la corte interamericana de derechos humanos, 2016, Tesis Doctoral dirigida por José Luis Piñar Mañas. España: Universidad CEU San Pablo. Disponible en: https://dialnet.unirioja.es/servlet/tesis? codigo=118416

Hernández-Torres, Francisco, La calidad de la atención a la salud en México a través de sus instituciones: 12 años de experiencia, Martha Alicia Alcántara Balderas (coord.), Secretaría de Salud, México, 2012. 
Híjar-Medina, Martha, María Victoria López-López, and Julia Blanco-Muñoz. "La violencia y sus repercusiones en la salud: Reflexiones teóricas y magnitud del problema en México." Salud pública de México 39 (1997): 565-572.

IbáÑez, Alejandro Acevedo, and Alba Florencia A. López Martín. El proceso de la entrevista: conceptos y modelos, Limusa, 2004.

IMSS. Comunicado del IMSS. "Médicos salvan a bebé tras realizar cesárea a su mamá en estado de coma". W Radio, ciudad de México, 17 de junio de 2019. Disponible en: https://wradio. com.mx/radio/2019/06/17/nacional/1560799796_173288. html

IMSS. Comunicado oficial No. 106/2019 "IMSS denuncia robo de medicamentos controlados" 2 de mayo de 2019 Instituto Mexicano del Seguro Social, México, 2019. Disponible en: http://imss.gob.mx/prensa/archivo/201905/106

Ley General de Acceso a las Mujeres a una Vida Libre de Violencia. LEY General de Salud.

Matías, Pedro, "Irma, la indígena mazateca que parió en el patio de un hospital", reportaje especial, en revista Proceso, México, Oaxaca, 4 de octubre de 2013, Disponible en: https://www. proceso.com.mx/reportajes/2013/10/4/irma-la-indigena-mazateca-que-pario-en-el-patio-de-un-hospital-124238. html

Mejía-Mejía, Elías, Metodología de la investigación cientifica, Lima, Universidad Nacional Mayor de San Marcos, 2005.

Muñoz-Rocha, Carlos, Metodología de la investigación, Oxford, México, 2015

NÁjar, Alberto, "Violencia en México: cómo se explica el nuevo récord en el número de homicidios", en BBC News Mundo, México, 22 julio 2019. Disponible en: https://www.bbc.com/ mundo/noticias-america-latina-49079323 
OMS. Constitución de la Organización Mundial de la Salud. Disponible en: https://www.who.int/governance/eb/constitution/es/

Ponce de León-Armenta, Metodología del derecho, México, Porrúa, 2011

Ramírez-Arias, José Luis, Federico Rodríguez Weber, and Raquel Ocampo Lujano. "La educación médica para las últimas generaciones." Acta médica Grupo Ángeles 16.3 (2018): 267-270.

Recomendación 03/2019, "Violaciones a los derechos humanos a la protección de la salud, a una vida libre de violencia obstétrica por inadecuada atención médica y acceso a la información en materia de salud, derecho a la vida y al principio del interés superior de la niñez”, CNDH, Baja California Sur, 2019.

RECOMENDACión GENERAL 31/2017 "Sobre la violencia obstétrica en el sistema nacional de salud", CNDH, Oaxaca, 2017

Recomendación No. 1/2014 "Sobre el caso de la inadecuada atención médica de $\mathrm{V} 1$ y su recién nacido $\mathrm{V} 2$, indígenas mazatecos, en el centro de salud rural del municipio San Felipe Jalapa de Díaz", Oaxaca, CNDH, 2014

Recomendaciones Internacionales a México en materia de Derechos Humanos. Disponible en: http://recomendacionesdh. $\mathrm{mx} /$

Salazar-Ugarte, Pedro y et al., "Las obligaciones del Estado", en La reforma constitucional sobre derechos humanos. Una guía conceptual, Instituto Belisario Domínguez, Senado de la República, Ciudad de México, 2014

Schmelkes, Corina y Nora Schmelkes, Manual para la presentación de anteproyectos e informes de Investigación, Oxford, tercera edición, México, 2012.

SSA. "Guía de Implantación. Modelo de atención a las mujeres durante el embarazo, parto y puerperio. Enfoque Humanizado, Intercultural y Seguro", SSA: México, 2012. 
SSA. Comunicado de Gobierno de la República, "Inicia fase 2 por coronavirus COVID-19", Ciudad de México, 2019, Disponible en: https://www.gob.mx/salud/ prensa/095-inicia-fase-2-por-coronavirus-covid-19

Tesis P.VII/2016 (10 ${ }^{\mathrm{a}}$ ), p.255. Gaceta del Seminario Judicial de la Federación. Tesis aislada. Décima época. Registro 2012597. Pleno. Libro 34, septiembre 2016. Tomo I. Materia Constitucional.

Tesis: I.6o.P.42 P (10a.), p.2310. Gaceta del Semanario Judicial de la Federación. Tesis: Aislada, décima época. Registro: 2005489. Sexto Tribunal Colegiado En Materia Penal del Primer Circuito, libro 3, febrero de 2014, Tomo III.

Tesis: P. LXV/2009, página 8. Semanario Judicial de la Federación y su Gaceta, Tomo XXX, diciembre de 2009, tesis: Aislada, novena época, registro 165813, Pleno en materia constitucional

Trilla, Antoni, et al., “Qué es el Coronavirus SARS-CoV-2?”, Clinic, Barcelona: 2020. Disponible en: https://www.clinicbarcelona.org/asistencia/enfermedades/covid-19/definicion

VÁzQuez, Luis Daniel y Sandra Serrano. "Los principios de universalidad, interdependencia, indivisibilidad y progresividad. Apuntes para su aplicación práctica”, en La reforma constitucional de derechos humanos: un nuevo paradigma, Pedro Salazar Ugarte y Miguel Carbonell Sánchez, (coords.) Instituto de Investigaciones Jurídicas, UNAM, México, 2011.

VIDEo del Gobierno de México. \#IMSS en ruinas: German Martínez, revista panorama, México, 2019. Ver en Línea. URL: https:// youtu.be/778-8nj89Rc /

VIDEO grabado por la paciente y difundido en redes. "Empleada del IMSS niega insulina a paciente”. AM noticias. Disponible en: https: / / www.youtube.com/watch?v=J66SczShp_U 
ENTREVISTAS 
